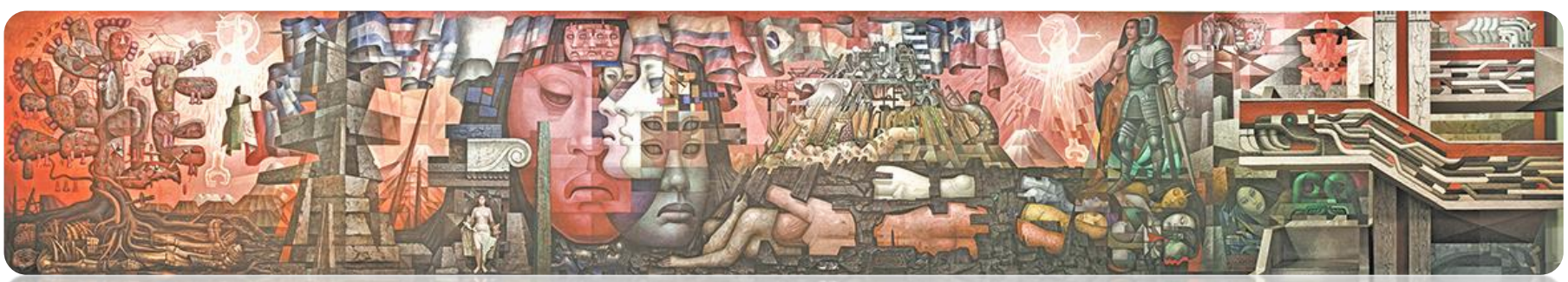

Rev. Hist., N²8, vol. 2, Julio-Diciembre 2021: 85-106

ISSN 0717-8832

https://doi.org/10.29393/RH28-28DOEC30028

\title{
Discutiendo sobre Bernardo O’Higgins. Formación ciudadana para el liderazgo en el Bicentenario*
}

\section{Deliberating about Bernardo O'Higgins. Citizenship education and leadership in the Bicentennial}

\author{
Eduardo Cavieres - Fernández* \\ Gabriel Villalón Gálvez** \\ Gabriela Vásquez Leyton***
}

\section{RESUMEN}

El objetivo de este trabajo es revisar si los antecedentes que se entregan a los estudiantes durante su enseñanza escolar respecto de Bernardo O'Higgins podrían servir para discutir acerca del liderazgo político tal como se da en la sociedad actual. Para ello, tomamos como referencia los contenidos fundamentales referidos a O'Higgins que se han incorporado en los textos escolares chilenos en los últimos quince años y, los vinculamos con aquellos elementos presentes en las directrices sobre Formación Ciudadana que son relevantes para la problemática del liderazgo en Chile. A partir de ello, subrayamos tres perspectivas que estos contenidos podrían aportar a la deliberación de los estudiantes en su preparación como ciudadanos.

Palabras claves: Bernardo O'Higgins, Formación Ciudadana, Liderazgo político, Enseñanza de la Historia, Deliberación en el aula, Textos escolares

\footnotetext{
* Esta investigación ha sido realizada gracias al financiamiento de FONDECYT (Fondo Nacional de Desarrollo Científico y Tecnológico) proyecto N. ${ }^{\circ} 1180475$.

* Universidad de Playa Ancha, Doctor en Educación. ORCID: https://orcid.org/0000-0003-3199-5090. Correo electrónico: ecavieres-cea@upla.cl.

** Universidad de Chile, Doctor en Didáctica de la Historia, la Geografía y las Ciencias Sociales. ORCID: https://orcid.org/0000-0003-0580-1870. Correo electrónico: gabriel.villalon@uchile.cl

**** Universidad Andrés Bello, Doctora en Didáctica de las Ciencias Sociales. ORCID: https://orcid.org/0000-00025633-7572. Correo electrónico: gabriela.vasquez@unab.cl
} 


\begin{abstract}
The goal of this paper is to analyze whether the background information about Bernardo O'Higgins provided to students during their school education could be used to discuss political leadership as it occurs in today's society. To this end, we take as a reference the fundamental contents referring to O'Higgins that have been incorporated in Chilean school textbooks in the last fifteen years, and we connect them with those elements present in the problem of leadership in Chile. From this, we highlight three perspectives that these contents could contribute to the deliberation of students in their preparation as citizens.
\end{abstract}

Key words: Bernardo O’Higgins, Citizenship education, Political leadership, History teaching, Classroom deliberation, School textbooks

Recibido: septiembre 2021

Aceptado: diciembre 2021

\title{
Introducción
}

A la luz de los nuevos debates y orientaciones para la formación ciudadana escolar, se hace necesario discutir sobre la pertinencia de enseñar, o no, la figura de Bernardo O'Higgins. En efecto, proponer como contenido de enseñanza a este personaje resulta, al menos en principio, contra-intuitivo respecto de una serie de criterios que adquieren protagonismo en las actuales formas de entender la vinculación entre la enseñanza de la historia con la formación ciudadana ${ }^{1}$. Consecuentemente, un mayor énfasis en la comprensión de los procesos por sobre un enfoque centrado en fechas y personajes individuales; el énfasis en el desarrollo de habilidades críticas que contrasten distintas visiones acerca del pasado para comprender el mundo de hoy sin aferrarse a una única interpretación dominante; así como una mayor visibilización de la participación que tuvieron los diversos grupos sociales en la conformación de la sociedad para alejarse de aproximaciones basadas en el elitismo político, son todos argumentos para que el estudio sobre O'Higgins sea menos relevante para la formación de ciudadanos. En ello también incide que, en la propia tradición nacional, O’Higgins haya sido parte de un panteón al servicio de las narrativas del Estado nación para imponer una forma de liderazgo político centrado en la élite y excluyente de vastos sectores de la población ${ }^{2}$.

\footnotetext{
${ }^{1}$ Ministerio de Educación, Bases curriculares. $7^{\circ}$ básico a $2^{\circ}$ medio, 2015.

${ }^{2}$ González, Fabian, Cerri, Luis y Rosso, Ademir. 2016. "Héroes y cultura histórica entre estudiantes en Chile", en Revista Brasileira de Historia, Vol. 36, N71, Sao Paulo, pp. 2 - 24.
} 
No obstante, nuestra intención no es argumentar a favor del estudio de Bernardo O’Higgins en vistas a refrendar el carácter institucional de su figura, sino analizar qué aspectos de su vida podrían aportar elementos para estudiantes que se incorporarán al mundo social y político cumpliendo tareas como ciudadanos. Específicamente, quisiéramos proponer una lectura acerca de los contenidos sobre Bernardo O'Higgins que se enseñan en el sistema escolar, para que su discusión o reflexión, por parte de los estudiantes, les aporten nuevas perspectivas para entender el liderazgo político, en el contexto, además, de las discusiones que al respecto se dan al interior de la ciudadanía. Estas discusiones constituyen una herramienta fundamental en el aula para que los estudiantes se formen en la deliberación en torno a temas controversiales $^{3}$. Estos temas remiten a conflictos públicos que suscitan una multiplicidad de perspectivas contrapuestas. Al respecto, el área curricular de la historia, precisamente, propone una serie de temáticas en torno a eventos y personajes, que no tan sólo fueron en sí mismos controversiales, sino que, además, generaron efectos históricos cuyas consecuencias aún generan antagonismo, al suscitar distintos posicionamientos respecto de cómo afectan valores fundamentales para la sociedad como la justicia o la igualdad.

Por cierto, la figura y el legado de Bernardo O’Higgins han sido controversiales en Chile en la medida que, históricamente, los distintos bloques políticos han tratado de adaptarlo a sus propios itinerarios ideológicos ${ }^{4}$. No obstante, nuestro objetivo es más bien revisar si los antecedentes que se entregan a los estudiantes respecto de Bernardo O'Higgins pudiesen servir para discutir acerca del liderazgo político tal como se da en la sociedad actual. Para ello, tomamos como referencia los contenidos fundamentales referidos a O'Higgins que se han incorporado en los textos escolares en los últimos 15 años, aunque han sido, más bien, secundarios e, incluso, marginales. Esto pudo haberse debido a los nuevos énfasis en la enseñanza de la historia mencionados anteriormente y que pueden haber influido en la elaboración de estos textos ${ }^{5}$. Por lo tanto, no se trata de analizar los contenidos de los textos escolares sobre O'Higgins, si son adecuados o no, sino de intentar establecer alguna vinculación entre ellos y aquellos elementos presentes en las directrices sobre Formación Ciudadana en Chile que son relevantes para la problemática discutida en este trabajo.

Teniendo a la vista estos antecedentes, a continuación, situamos este análisis bajo un contexto teórico que presente el fenómeno del liderazgo en la sociedad; y en relación a los desafíos que existen en el ámbito de la enseñanza de la historia que suponen su vinculación a temáticas de relevancia pública. Luego, proponemos tres ámbitos de reflexión: el tema del

\footnotetext{
${ }^{3}$ McAvoy, Paula y Hess, Diana. 2013. "Classroom deliberation in an era of political polarization", Curriculum Inquiry, Vol. 43, N 1 , Toronto, pp. 14 - 47.

4 Guerrero, Cristian y Cárcamo, Ulises. 2013. “Bernardo O’Higgins, entre izquierda y derecha. Su figura y legado en Chile: 1970-2008", en Cuadernos de Historia, Vol. 39, Santiago, pp. 113 - 146.

${ }^{5}$ Romero, Luis Alberto. 2009. "Enfrentar al enano nacionalista: una mirada a los libros de texto", Temas de Historia Argentina y Americana, Vol. 14, Buenos Aires, pp. 197 - 214.
} 
liderazgo en la formación ciudadana; la dimensión valórica en el liderazgo político; el liderazgo ciudadano con orientación global. Para hacerlo, en cada ámbito, revisamos críticamente algunos elementos de las directrices de Formación Ciudadana, señalando algunos vacíos presentes en ellas, y ante los cuales, sugerimos algunos contenidos de la vida de O'Higgins que podrían ser discutidos para reforzar la formación ciudadana de los estudiantes.

\section{Liderazgos políticos y ciudadanía}

En este texto, el tema del liderazgo político es contextualizado por la relación que se establece entre Estado y ciudadanía, la que supone, por una parte, una institucionalidad desde donde se establece el gobierno político, y por otra, el consentimiento de la ciudadanía ${ }^{6}$. En virtud de ello, los Estados que se adscriben a un modelo democrático, han ido estableciendo mecanismos de sufragio para posibilitar niveles formales de participación por parte de la ciudadanía. No obstante, algunos análisis advierten que dichos mecanismos tienden a instalarse bajo el supuesto de que no todas las personas tienen las capacidades para cumplir roles públicos, pues, de hecho, la mayoría de los individuos debieran preocuparse más bien por sus asuntos privados, y, por tanto, que se debiese delegar a un grupo especializado -la mayor de las veces en la forma de partidos políticos- la representación de los intereses individuales y la toma de decisiones sobre los asuntos públicos ${ }^{7}$. Esta comprensión, continúan estos análisis, no sólo suscitaría bajos niveles de compromiso para que los ciudadanos se involucren más activamente en la construcción de un bien general a través de las distintas organizaciones sociales; sino también promovería un elitismo centrado en líderes políticos que, eventualmente, podrían favorecer solo a los grupos que ellos representan ${ }^{8}$.

Desde esa perspectiva, el tema del liderazgo político constituye una problemática medular en la conformación del sistema democrático tal como se ha ido concibiendo en los diversos países. Así, como lo han planteado múltiples autores, el fenómeno mismo de la creciente apatía de la ciudadanía por participar en eventos eleccionarios, particularmente entre los jóvenes, se ha ido asociando al rechazo creciente hacia los partidos políticos y sus líderes por su carácter poco representativo y su vinculación a intereses privados ${ }^{9}$. Más aún, esta desconexión entre representantes y representados ha adquirido connotaciones éticas cuando los líderes políticos han usado su poder para provecho personal apropiándose ilegalmente de

\footnotetext{
${ }^{6}$ Dahl, Robert. 1988. On democracy, New Haven, Yale University Press.

7 Dalton, Russel. 2014. Citizen politics: public opinion and political parties in advanced industrial societies, London, SAGE.

${ }^{8}$ McLaughlin, Terence. 1992. "Citizenship, diversity and education, a philosophical perspective", Journal of Moral Education, Vol. 21, N 3, Birmingham, pp. 235 - 250.

9 Mycock, Andrew y Tonge, Jonathan. 2012. "The party politics of youth citizenship and democratic engagement", Parliamentary Affairs, Vol. 65, London, pp. 138 - 161; Henn, Matt y Foard, Nick. 2014. "Social differentiation in young people's political participation: the impact of social and educational factors on youth political engagement in Britain", Journal of Youth Studies, Vol. 17, № 3, Londres, pp. 360 - 380.
} 
recursos económicos o utilizando indebidamente sus influencias ${ }^{10}$. Igualmente, en América Latina, en donde existe una tradición autoritaria de ejercicio del poder, se han preservado formas personalistas de liderazgo, a veces en la forma de bloques políticos cerrados, que permanentemente dañan los procesos de democratización y la institucionalidad democrática instalada en los países en la región, con el fin de perpetuar intereses políticos y económicos particulares, incluso utilizando la violencia política para suprimir cualquier forma de oposición y pluralismo ${ }^{11}$.

En Chile, el tema del liderazgo ha sido desarrollado por Araujo ${ }^{12}$ a través del concepto de autoridad. Desde su perspectiva, ésta constituye una problemática transversal que resulta del permanente miedo a la supuesta incapacidad de obedecer que tendrían los subordinados, y al peligro de caos que aquello produciría. Dicho temor ha incidido en dos arquetipos de ejercicio de la autoridad que han permanecido en el tiempo en el país. Mientras uno de ellos alude al tipo de relación que se estableció en la hacienda rural, en donde el patrón estableció una autoridad jerárquica directa y personal sobre los peones, con el uso abusivo de la fuerza para conseguir el control y disciplinamiento; el otro se desarrolló para dar respuesta a la inestabilidad social y política que se generó tras el proceso de Independencia -y, en la cual, a Bernardo O’Higgins se le atribuyó parte de la responsabilidad- estableciéndose un poder central, el Estado, al que se le atribuyó la prerrogativa de decidir unilateralmente sobre los asuntos de interés nacional, incluso utilizando mecanismos excepcionales de represión. Actualmente, ante nuevas formas de democratización y empoderamiento individual de los ciudadanos, estos arquetipos estarían entrado en crisis dado su autoritarismo y su incapacidad de crear adhesión a los mandatos más allá de la obediencia externa.

Aplicado al ámbito escolar en el país, este conjunto de aspectos referidos al tema del liderazgo público ha quedado reflejado en el trabajo de Muñoz-Labraña, Martínez-Rodríguez y Muñoz-Grandón ${ }^{13}$ sobre las percepciones de estudiantes de último año de enseñanza media en Chile acerca de los políticos, su relación con el poder, y su distancia respecto de los intereses de la ciudadanía. Este trabajo también incluye las percepciones que tienen los estudiantes respecto de los cambios que debieran darse para que el liderazgo político tuviese relevancia para la ciudadanía, en referencia a dos ámbitos: a) el establecimiento de regulaciones y normas que obliguen a los líderes políticos a tener más empatía, se aferren menos al poder, y tengan un estilo de vida (económica) más cercana al común de los ciudadanos; b) que los mismos

\footnotetext{
${ }^{10}$ Stoker, Gerry. 2006. Why democracy matters. Making democracy work, New York, Palgrave MacMillan.

${ }^{11}$ Smith, Peter. 2012. Democracy in Latin America. Political change in comparative perspective, New York, Oxford University Press.

12 Araujo, Kathya. 2016. El miedo a los subordinados. Una teoría de la autoridad, Santiago de Chile, LOM.

13 Muñoz-Labraña, Carlos, Martínez-Rodríguez, Rosendo y Muñoz-Grandón, Carlos. 2016. "Percepciones del estudiantado sobre la política, los partidos políticos y las personas dedicadas a la política al finalizar la educación secundaria en Chile", en Revista Electrónica Educare, Vol. 20, № 1, Heredia, pp. 1 - 16.
} 
jóvenes ingresen a la vida política para reemplazar a los dirigentes actuales con modos de liderazgo distintos. Esta contribución, no sólo es relevante porque ahonda en una temática poco explorada por la literatura en Chile, sino que, además, abre las interrogantes que mueven al presente texto respecto a la necesidad de aportar elementos para que los estudiantes discutan sobre el liderazgo ante las demandas sociales que permanentemente se suscitan en el país.

\section{Enseñanza de la historia, formación ciudadana y el liderazgo como tema deliberativo}

Si bien la relación entre la enseñanza de la historia escolar y la formación ciudadana ha sido largamente establecida, en las últimas décadas, ha incluido un viraje desde un enfoque centrado en el desarrollo de un conocimiento histórico vinculado al interés del Estado nación, a otro que pone su foco en el análisis crítico del pasado a través de la formación del pensamiento histórico en vinculación con las actuales problemáticas políticas, económicas y sociales ${ }^{14}$. La primera de estas tradiciones coloca el énfasis en la adhesión a los proyectos nacionales tal como fueron concebidos por los Estados. Su finalidad es transmitir un relato a los estudiantes que les permita identificarse con el proyecto común de la nación, lo que, a su vez, exige la enseñanza de eventos y personajes considerados fundamentales para la construcción de la patria. Esta aproximación enfatiza la formación de una ciudadanía responsable, con un énfasis fundamental en la participación representativa y en los derechos y deberes políticos que dan estabilidad a la institucionalidad del Estado, y en la construcción de una identidad nacional de tipo homogénea que todos los ciudadanos debiesen defender y promover ${ }^{15}$.

Por contrapartida, un enfoque alternativo para la enseñanza de la Historia se centra en las habilidades de los estudiantes para comprender críticamente el pasado a través de la revisión de fuentes, propia de la investigación histórica, y establecer una mayor vinculación entre conocimiento histórico y la comprensión de las problemáticas que caracterizan a la vida en sociedad ${ }^{16}$. En términos de la formación ciudadana, las habilidades de análisis histórico que

\footnotetext{
${ }^{14}$ Carretero, Mario. 2007. Documentos de Identidad. La construcción de la memoria histórica en un mundo global, Buenos Aires, Paidós; Villalón-Gálvez, Gabriel. 2021. “Conocimiento histórico y formación ciudadana. Inclusión de otras narrativas históricas para una Nueva Historia Escolar", en REIDICS. Revista De Investigación En Didáctica De Las Ciencias Sociales, №8, Cáceres, pp. 89 - 105.

15 Villalón-Gálvez, Gabriel, Cavieres-Fernández, Eduardo y Vásquez-Leyton, Gabriela. 2020. "Vinculando la enseñanza de la independencia nacional con temas socialmente relevantes para la formación de ciudadanos", en Sophia Austral, №26, Punta Arenas, pp. 33 - 50.

16 Lévesque, Stephane y Clark, Penney. 2018. "Historical thinking: Definitions and educational applications", en Metzger, Scott. A. y Harris, Lauren M. (Eds.) The Wiley International Handbook of history teaching and learning, New York, Willey Blackwell, pp. 117 - 148; Van Boxtel, Carla y van Drie, Jannet. 2018. "Historical reasoning: Conceptualizations and educational applications", en Metzger, Scott. A. y Harris, Lauren M. (Eds.) The Wiley International Handbook of history teaching and learning, New York, Willey Blackwell, pp. 149-176.
} 
adquieren los estudiantes les permite desarrollarse como ciudadanos informados, capacitados para decodificar la información que reciben desde la esfera pública y tomar decisiones que favorezcan a la democracia dentro de los entornos en que se desenvuelven ${ }^{17}$. Ello incluye cuestionar la distribución de poder en la sociedad y la desigualdad que se establece entre los grupos sociales; posicionarse frente a los conflictos que resultan de la relación Estado y ciudadanía; y asumir el protagonismo en la expansión de principios tan fundamentales como la libertad y la justicia ${ }^{18}$.

Un aspecto metodológico esencial de esta perspectiva de enseñanza de la Historia, es la incorporación de temas controversiales y de contenidos históricos que permitan a los estudiantes conocer otras narrativas históricas, no presentes de manera tradicional en el currículo escolar ${ }^{19}$. Dicha aproximación, permite el desarrollo de habilidades propias del conocimiento histórico y de la formación ciudadana, generando en los estudiantes un acercamiento crítico tanto al análisis del pasado como a las problemáticas públicas del presente a través de la discusión y/o deliberación ${ }^{20}$. Ello incluye el análisis respecto de las decisiones y acciones de las personas en el pasado y evaluar sus consecuencias para el presente $^{21}$; lo que supone comprenderlas dentro de las coordenadas temporales y los contextos sociales de su época que explican los conflictos de intereses o de valores entre grupos que incidieron, por ejemplo, en la actuación de líderes políticos cuyos disposiciones aún tienen influencia en el presente ${ }^{22}$.

\footnotetext{
${ }^{17}$ Endacott, Jason, Dingler, Matt y O'Brien, Joe. 2020. "To What Purpose? The ends and means of history education in the modern world", en Berg, Christopher, y Christou, Theodore (Eds.) The Palgrave Handbook of History and Social Studies Education, Cham, Springer, pp. $541-573$.

18 Ross, E. Wayne. 2017. Rethinking Social Studies: Critical Pedagogy in Pursuit of Dangerous Citizenship, Charlotte, Information Age Publishing, Inc.

${ }^{19}$ Nordgren, Kenneth y Johansson, Maria. 2015. "Intercultural historical learning: A conceptual framework", Journal of Curriculum Studies, Vol. 47, N¹, Londres, pp. 1 - 25; Goldberg, Tsafrir y Savenije, Geerte. 2018. "Teaching controversial historical issues", en Metzger, Scott y Harris, Lauren M. (Eds.) The Wiley International Handbook of history teaching and learning, New York, Willey Blackwell, pp. $503-526$.

${ }^{20}$ McAvoy, Paula y Hess, Diana. 2013. "Classroom deliberation"; Cavieres-Fernández, Eduardo y Uzcátegui, Ramón. 2021. "Problemáticas públicas como temáticas controversiales. Profesorado de Historia y la enseñanza de la Independencia Nacional y de Formación Ciudadana en el Bicentenario de Chile", en Revista Electrónica Educare, Vol. 25, N. 2, Costa Rica, pp. 1 - 21.

${ }^{21}$ Seixas, Peter. 2017. "Historical consciousness and historical thinking", en Carretero, Mario, Berger, Stefan y Grever, Maria (Eds.) Palgrave handbook of research in historical culture and education, Londres, Palgrave Macmillan, pp. $59-72$.

22 Wineburg, Sam. 2001. Historical Thinking and other unnatural acts. Charting the future of teaching the past, Filadelfia, Temple University Press; Lévesque, Stephane. 2008. Thinking historically: Educating students for the twenty-first century, Toronto, University of Toronto Press.
} 


\section{Bernardo O’Higgins en la enseñanza escolar y la Formación Ciudadana}

Por cierto, no es posible delimitar específicamente los contenidos que se enseñan en el ámbito escolar sobre Bernardo O'Higgins, considerando la autonomía de los profesores para tomar decisiones al respecto. No obstante, los textos escolares, al cumplir su rol de oficializar lo que debe transmitirse a los estudiantes, han fijado algunas referencias a la vida de O'Higgins que podrían tener implicancias para discutir la problemática del liderazgo en el contexto de las políticas de Formación Ciudadana en el país. Específicamente, en este trabajo, consideramos los principales textos escolares de Historia y Cs. Sociales, de enseñanza media, publicados en Chile a partir del año 2000, y que incluyen la unidad de la Independencia Nacional. En la tablas siguientes se presentan sintéticamente las referencias que incluyen estos textos, en dicha unidad, respecto de Bernardo O'Higgins.

Tabla 1

Bernardo O'Higgins en los textos escolares (1)

\begin{tabular}{|c|c|c|c|c|c|c|}
\hline Texto & Abdicación de O`Higgins & \multicolumn{5}{|c|}{ Proclamación de la Independencia } \\
\hline 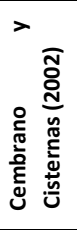 & $\begin{array}{l}\text { (línea de tiempo) } \\
\text { Mención a la Abdicación } \\
\text { de O`Higgins } \\
\text { (p. 101) }\end{array}$ & \multicolumn{5}{|c|}{$\begin{array}{l}\text { (...) de hacer saber a la gran confederación del género humano que el territorio continental } \\
\text { de Chile y sus islas adyacentes, forman de hecho y por derecho un Estado libre, } \\
\text { independiente y soberano, y quedan para siempre separados de la Monarquía de España, } \\
\text { con plena aptitud de adoptar la forma de gobierno que más convenga a sus intereses. } \\
\text { Bernardo O`Higgins Riquelme, Concepción, 10 de enero } 1818 \text { (Fuente histórica escrita: } \\
\text { Proclamación de la Independencia, fragmento) (p. 100) }\end{array}$} \\
\hline \multirow[b]{2}{*}{ 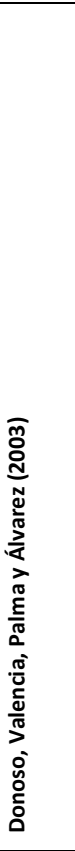 } & \multicolumn{2}{|l|}{ Gobierno } & $\begin{array}{l}\text { Empréstito de } \\
\text { Inglaterra }\end{array}$ & Ejército Libertador & $\begin{array}{l}\text { Relación con los } \\
\text { "héroes de la } \\
\text { Independencia" }\end{array}$ & $\begin{array}{l}\text { Proclamación de } \\
\text { la Independencia }\end{array}$ \\
\hline & \multicolumn{2}{|c|}{$\begin{array}{ll}\text { - } & \text { Declaración de Independencia (1818) } \\
\text { - } & \text { Dictación de las Constituciones de } \\
& 1818 \text { y } 1822 \\
\text { - } & \text { Abolición de los títulos de nobleza } \\
\text { - } \quad \text { Creación de la Legión al Mérito, la } \\
\text { Escuela Militar (1817) y la Armada } \\
\text { (1818) } \\
\text { - } \quad \text { Recuperación de Valdivia y } \\
\text { expedición Libertadora al Perú } \\
\text { (1820) } \\
\text { - Publicación de La Gaceta del } \\
\text { Gobierno de Chile } \\
\text { - Reapertura del Instituto y Biblioteca } \\
\text { Nacional } \\
\text { Creación Mercado de Abastos, } \\
\text { Cementerio General } \\
\text { - Construcción del paseo público de La } \\
\text { Cañada } \\
\text { Creación de Cementerio de } \\
\text { Disidentes de Valparaíso } \\
\text { Fundación de Vicuña, La Unión y San } \\
\text { Bernardo (p. 112) }\end{array}$} & $\begin{array}{l}\text { Gobierno de } \\
\text { Bernardo } \\
\text { O`Higgins } \\
\text { (1817-1823): } \\
\text { Misiones } \\
\text { diplomáticas a } \\
\text { Santa Sede y a } \\
\text { Gran Bretaña } \\
\text { (p.112) }\end{array}$ & $\begin{array}{l}\text { La Batalla de } \\
\text { Chacabuco, el } 12 \\
\text { de febrero de } \\
\text { 1817, permitió al } \\
\text { Ejército } \\
\text { Libertador de los } \\
\text { Andes, liderado } \\
\text { por Bernardo } \\
\text { O`Higgins y José } \\
\text { de San Martín, } \\
\text { recuperar el } \\
\text { gobierno de } \\
\text { Santiago. } \\
\text { (p. 123) }\end{array}$ & $\begin{array}{l}\text { "Si tenemos } \\
\text { que recordar } \\
\text { algún } \\
\text { personaje, } \\
\text { seguramente } \\
\text { los nombres } \\
\text { de Bernardo } \\
\text { O`Higgins, } \\
\text { José Miguel } \\
\text { Carrera y } \\
\text { Manuel } \\
\text { Rodríguez se } \\
\text { repetirán con } \\
\text { frecuencia”. } \\
\text { (p. 110) }\end{array}$ & $\begin{array}{l}\text { Gobierno de } \\
\text { Bernardo } \\
\text { O`Higgins } \\
\text { (1817-1823): } \\
\text { Declaración de } \\
\text { Independencia } \\
\text { (p. 112) } \\
\text { Tras la victoria } \\
\text { en Maipú (5 de } \\
\text { abril de 1818) } \\
\text { los españoles } \\
\text { eran expulsados } \\
\text { de la zona } \\
\text { central. } \\
\text { (p. 123) }\end{array}$ \\
\hline
\end{tabular}

Fuente: elaboración propia 
Tabla 2 Bernardo O'Higgins en los textos escolares (2)

\begin{tabular}{|c|c|c|c|c|c|c|c|}
\hline \multirow[b]{2}{*}{ 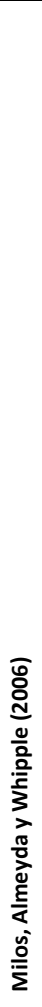 } & \multicolumn{2}{|l|}{ Liderazgo/Autoridad } & Gobierno & \multicolumn{2}{|c|}{$\begin{array}{l}\text { Ejército Libertador } \\
\text { Expedición Libertadora al } \\
\text { Perú }\end{array}$} & $\begin{array}{l}\text { Relación con los } \\
\text { "héroes de la } \\
\text { Independencia" }\end{array}$ & $\begin{array}{l}\text { Proclamación } \\
\text { Independencia }\end{array}$ \\
\hline & \multicolumn{2}{|c|}{$\begin{array}{l}\text { Las medidas de O’Higgins le } \\
\text { significaron diferencias con } \\
\text { la élite local que respetaban } \\
\text { sus principios liberales, pero } \\
\text { les afectaban sus intereses. } \\
\text { Aceptaban su personalismo } \\
\text { y autoritarismo sólo cuando } \\
\text { existía riesgo militar. Esta } \\
\text { desconfianza hacia O'Higgins } \\
\text { se profundizó con la } \\
\text { Constitución de 1822, que lo } \\
\text { designaba Director Supremo } \\
\text { con la facultad de nombrar } \\
\text { autoridades locales y } \\
\text { provinciales, y a los } \\
\text { miembros del Senado. En } \\
\text { enero de } 1823 \text {, los } \\
\text { principales vecinos de } \\
\text { Santiago se reunieron en } \\
\text { Cabildo Abierto y O'Higgins } \\
\text { fue conminado a renunciar a } \\
\text { su cargo. Para evitar una } \\
\text { guerra civil, partió al exilio. } \\
\text { (p.105) }\end{array}$} & $\begin{array}{l}\text { O`Higgins es } \\
\text { designado Director } \\
\text { Supremo del nuevo } \\
\text { gobierno. Proclamó la } \\
\text { Independencia. } \\
\text { (p.104) } \\
\text { Creó la Escuela Militar } \\
\text { y la Armada de Chile. } \\
\text { Mejoró la estructura } \\
\text { urbana de Santiago } \\
\text { (Mercado de Abastos, } \\
\text { el paseo de la Cañada } \\
\text { y el Cementerio } \\
\text { General). Abolió los } \\
\text { títulos de nobleza y el } \\
\text { uso de escudos de } \\
\text { armas. Prohibió los } \\
\text { juegos de azar, peleas } \\
\text { de gallo y corridas de } \\
\text { toros). } \\
\text { (p. 105) }\end{array}$ & \multicolumn{3}{|c|}{ 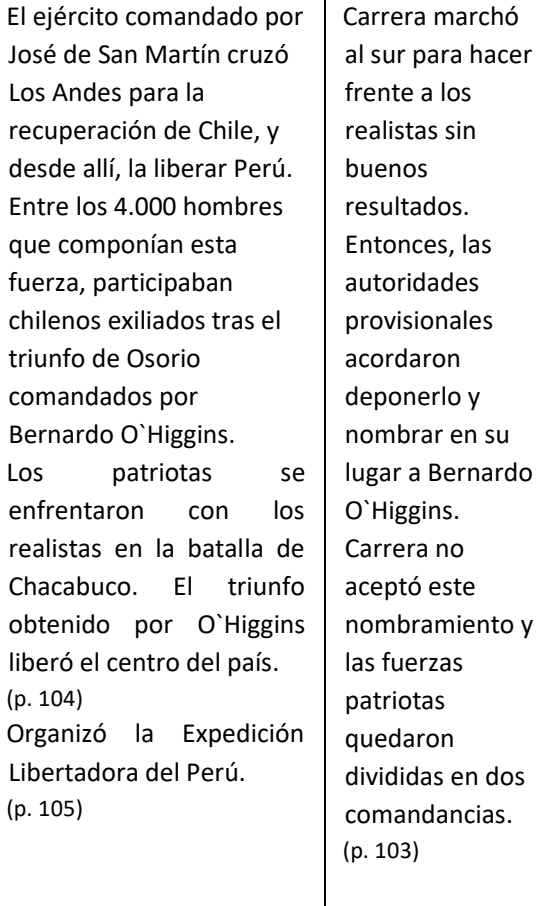 } & $\begin{array}{l}\text { Se designó a } \\
\text { O`Higgins } \\
\text { como Director } \\
\text { Supremo para } \\
\text { organizar el } \\
\text { gobierno. } \\
\text { Proclamó } \\
\text { solemnemente } \\
\text { la } \\
\text { Independencia } \\
\text { de Chile el } 12 \\
\text { de febrero de } \\
\text { 1818. } \\
\text { (p.105) }\end{array}$ \\
\hline \multirow[b]{2}{*}{ 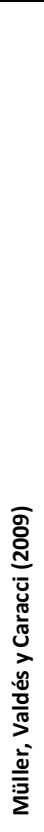 } & Liderazgo / Autoridad & \multicolumn{2}{|c|}{ Gobierno } & & \multicolumn{3}{|c|}{ o Libertador / Expedicio } \\
\hline & $\begin{array}{l}\text { A pesar del importante } \\
\text { aporte de O' Higgins en } \\
\text { esta y otras materias, } \\
\text { su gobierno no se } \\
\text { alineó con las ideas de } \\
\text { la aristocracia chilena } \\
\text { de la época, ya que era } \\
\text { autoritario y tenía } \\
\text { ideas más liberales, las } \\
\text { que no fueron bien } \\
\text { recibidas por este } \\
\text { grupo, cada vez más } \\
\text { poderoso, por lo tanto, } \\
\text { debió abdicar y partir } \\
\text { al exilio en Perú. } \\
\text { (p.117) }\end{array}$ & \multicolumn{3}{|c|}{$\begin{array}{l}\text { Patria Nueva (1817-1823): Batalla de Chacabuco } \\
\text { hasta abdicación de Bernardo O’Higgins. } \\
\text { Gobierno de Bernardo O’ Higgins (1817-1823) } \\
\text { - Constituciones de } 1818 \text { y } 1822 . \\
\text { - Misiones diplomáticas a la Santa Sede y Gran } \\
\text { Bretaña. } \\
\text { - Estados Unidos reconoce la Independencia de } \\
\text { Chile. } \\
\text { - Abolición de los títulos de nobleza. } \\
\text { - Creación Legión del Mérito, Escuela Militar y } \\
\text { Armada. } \\
\text { - Publicación de La Gaceta del gobierno de } \\
\text { Chile. } \\
\text { - Reapertura del Instituto y la Biblioteca } \\
\text { Nacional. } \\
\text { - Creación del Mercado de Abastos y del } \\
\text { Cementerio General. } \\
\text { - Construcción paseo público la Alameda de las } \\
\text { Delicias. } \\
\text { - Creación del Cementerio de Disidentes de } \\
\text { Valparaíso. } \\
\text { - Fundación de Vicuña, La Unión y San } \\
\text { Bernardo. (p.107) }\end{array}$} & \multicolumn{3}{|c|}{$\begin{array}{l}\text { La Batalla de Chacabuco, el } 12 \text { de febrero } \\
\text { de } 1817 \text {, permitió al Ejército Libertador de } \\
\text { los Andes, liderado por Bernardo O’Higgins } \\
\text { y José de San Martín, recuperar el gobierno } \\
\text { de Santiago, dando inicio a la Patria Nueva } \\
\text { (1817-1823). En } 1818 \text { Chile proclamó su } \\
\text { independencia y luego de la victoria en } \\
\text { Maipú en abril de ese año, los españoles } \\
\text { fueron definitivamente expulsados de la } \\
\text { zona central consolidándose así la } \\
\text { independencia del país } \\
\text { (p. 117) }\end{array}$} \\
\hline
\end{tabular}

Fuente: elaboración propia 
A continuación, vinculamos estas referencias presentes en los textos escolares con las políticas oficiales sobre Formación Ciudadana que comenzaron a ser implementadas a partir del año 2004. Evidentemente, ni estos contenidos ni estas políticas constituyen un todo orgánico ni articulado, pero tomados en su conjunto, posibilitan la discusión en torno a tres grandes temas sobre el liderazgo que son coherentes con los análisis previamente desarrollados: a) el liderazgo político; b) la dimensión valórica en el liderazgo político; c) la dimensión global en el liderazgo político.

\section{El tema del poder y el liderazgo político en O'Higgins}

Las actuales políticas sobre Formación Ciudadana derivaron de la preocupación de los Estados por los crecientes niveles de apatía y de baja participación de los ciudadanos, de manera particular entre los jóvenes ${ }^{23}$. Consecuentemente, entre las estrategias para contrarrestar este efecto estuvo el resocializar a los estudiantes con los órganos de la institucionalidad política para aumentar su compromiso hacia la estabilidad del sistema político democrático. Desde esa perspectiva, sólo se tendió a promover un concepto de participación ciudadana más bien referido a procesos eleccionarios; y, se promovió la participación de los estudiantes en asociaciones locales, pero acentuándose el servicio y la deliberación consultiva, no resolutiva ${ }^{24}$. Tampoco, se dio importancia a que los jóvenes desarrollaran formas de liderazgos que moviesen a la participación más activa o a la creación de espacios más representativos para los propios estudiantes más allá de la institucionalidad política o de las formas institucionales reservadas para ellos. En ese sentido, tampoco se ofrecieron criterios para que los jóvenes pudiesen formarse para asumir en el futuro puestos de liderazgo, comprendiendo las dificultades y responsabilidades que aquello significa, además de anticipar los conflictos que emanan de las dinámicas de poder que se dan entre los grupos sociales.

Desde esa perspectiva, los contenidos presentes en los textos escolares sobre Bernardo O’Higgins aportan elementos para reflexionar respecto de la primera generación de líderes de las nacientes repúblicas latinoamericanas $y$, por tanto, de un grupo aristocrático monopolizador del poder, por ejemplo, a través de la redacción de constituciones personalistas $^{25}$. No obstante, aquello le significó a O’Higgins el tener que desarrollar habilidades importantes como líder a pesar de sus limitaciones; no tan sólo para asumir un rol

\footnotetext{
${ }^{23}$ Advisory Group on Citizenship. 1998. Education for citizenship and the teaching of democracy in schools, London, Qualifications and Curriculum Authority; Comisión Formación Ciudadana. 2004. Informe Comisión Formación Ciudadana, Santiago de Chile, Ministerio de Educación.

${ }^{24}$ Cavieres-Fernandez, Eduardo. 2019. "Urban high school students' experiences of participation as a counter-story constellation to institutional and policy guidelines in Chile", Urban Education, Washinton, pp. 1 - 12.

25 Donoso, María Loreto; Valencia, Lucía; Palma, Daniel y Álvarez, Rolando. 2003. Historia y Ciencias Sociales. $2^{\circ}$ Educación Media, Santiago de Chile, Santillana del Pacífico S.A. de Ediciones, p. 110; Mendizábal, María y Riffo, Jorge. 2013. Historia, Geografía y Ciencias Sociales. $2^{\circ}$ Medio Editorial. Texto del Estudiante, Santiago de Chile, Ediciones SM Chile S.A., p.110.
} 
militar frente al poder español sin tener la preparación para hacerlo; sino también cuando gobierna teniendo serias desavenencias con la aristocracia santiaguina, y en medio de un ambiente de desorden social resultante de la guerra por la independencia ${ }^{26}$. Ante ello, ciertamente, tuvo que crear y desarrollar alianzas a fin de poder asumir un rol protagónico en el proceso independentista ${ }^{27}$. De este modo, estudiar el liderazgo de O'Higgins no tan sólo permite discutir sobre la dinámica de la concentración del poder, y cómo aún está vigente en Chile, así como analizar sus limitaciones y beneficios, sino también sugerir otras preguntas importantes de discusión tales como: ¿Qué debería hacer una autoridad cuando entra en conflicto con grupos de elites que concentran el poder en el país? ¿Qué tipo de estrategias debería usar? ¿Qué tipo de alianzas debería construir?

Sin embargo, los modos de gobernar imperantes no pueden entenderse sino es desde la evolución que tuvo la nación y el Estado moderno y su efecto sobre la homogeneización política, económica y social ${ }^{28}$. Dicha complejidad, supone ciertos alcances y límites que los estudiantes debieran tomar en cuenta al reflexionar sobre los liderazgos actualmente presentes y que están, en parte, condicionados no sólo por los defectos del pasado, sino por las restricciones que derivan de las coordenadas históricas dentro de las cuales evolucionó el Estado moderno. Sin embargo, la contribución de O'Higgins también puede considerarse como un liderazgo bisagra en cuanto se ubica en un límite difuso entre un modo de gobernar vigente (de corte monárquico) y el incipiente modo de gobernar que se avizoraba (republicano). En ese sentido, si bien el estudio de O'Higgins sirve para discutir respecto de los déficits que derivan del Estado nación tales como el elitismo excluyente, y analizar posibles propuestas para resolverlos; también ofrece perspectivas al considerar la época de cambio que le tocó vivir y, en la cual, fue necesario pensar en términos más bien paradigmáticos sobre la ciudadanía y el liderazgo; tal como ocurre hoy cuando va surgiendo la necesidad de pensar en ciudadanías y liderazgos más bien post-nacionalistas ${ }^{29}$.

\footnotetext{
${ }^{26}$ Milos, Pedro; Almeyda, Liliam y Whipple, Pablo. 2006. Historia y Ciencias Sociales. $2^{\circ}$ Medio. Texto para el Estudiante, Santiago de Chile, MN Editorial Ltda., p.105; Müller, Javiera; Valdés, Magdalena y Caracci, María Soledad. 2009. Historia y Ciencias Sociales. $2^{\circ}$ Educación Media. Texto para el Estudiante, Santiago de Chile, Santillana del Pacífico S.A. de Ediciones, p.117.

27 Javiera Müller, Magdalena Valdés y María Soledad Caracci. p. 117; Landa del Rio, Luis y Pinto, Victoria. 2017. Historia, Geografía y Ciencias Sociales. $8^{\circ}$ básico. Texto del Estudiante, Santiago de Chile, Ediciones SM Chile S.A., p. 218.

${ }^{28}$ Heater, Dereck. 2004. Citizenship. The civic ideal in world history, politics and education, New York, Manchester University Press.

${ }^{29}$ Ní Mhurchú, Aoileann. 2014. "Citizenship beyond the state sovereignty", en Isin, Engin y Nyers, Peter (eds.). Routledge handbook of global citizenship studies, New York, Routledge.
} 


\section{La dimensión ética en el liderazgo político de O’Higgins}

Un contenido importante de la Formación Ciudadana en Chile se refiere al tema de las responsabilidades y de los derechos de los ciudadanos dentro del sistema político ${ }^{30}$. De manera particular, se ha enfatizado, dada la experiencia histórica de Chile, el preparar a los ciudadanos para la defensa de los derechos humanos. En ello, se pueden desprender dos formas de aproximarse frente al tema. Por un lado, desde una aproximación institucional procedimental. Puesto que el foco está puesto sobre el empoderamiento de la ciudadanía para evitar abusos por parte de los órganos del Estado, una forma de promover el respeto a los derechos de las personas es delimitarlo dentro de esa relación. Por otro lado, desde una aproximación más ligada a una formación que dé a los estudiantes criterios éticos para tomar decisiones más adecuadas para fomentar la convivencia social y el bien común. No obstante, en las directrices sobre Formación Ciudadana es más evidente la primera aproximación y, por tanto, se abre la discusión respecto de si no sería también necesario vincular, de manera más estrecha, lo político con lo valórico - ético en el desarrollo de futuros líderes tanto para la sociedad civil como para la institucionalidad política, no como esferas separadas, sino en recíproca correspondencia.

Si bien el contexto político en el cual se desarrolló el liderazgo de O’Higgins dista sustancialmente del actual, en los contenidos presentes en los textos escolares, es posible encontrar similitudes entre ciertas ideas ilustradas y liberales presentes en el proyecto de O'Higgins y aquellas que posteriormente establecieron el horizonte ético de la institucionalidad política y civil en Chile ${ }^{31}$. Siguiendo estas ideas, O’Higgins desarrolló un liderazgo autoritario y se involucró en pugnas con opositores a fin de imponer su comprensión del orden social ${ }^{32}$. Sin embargo, al mismo tiempo, estas ideas resaltan su talante valórico cuando renuncia al poder en pos de la mayor unidad nacional o cuando, a contrapelo de su estabilidad como gobernante, decide abolir determinados derechos de la élite chilena a fin de promover la igualdad en la república naciente ${ }^{33}$. También inspiran las obras que dirigió para promover la instrucción pública y el mejoramiento urbano ${ }^{34}$. Por tanto, todo aquello permite discutir respecto de las implicancias éticas que tienen las ideas políticas para sustentar modos

\footnotetext{
30 División de Educación General. 2016. Orientaciones para la elaboración del plan de formación ciudadana, Santiago de Chile, Ministerio de Educación.

${ }^{31}$ Müller, Javiera; Valdés, Magdalena y Caracci, María Soledad. 2009. Historia y Ciencias Sociales, p.117; Milos, Pedro; Almeyda, Liliam y Whipple, Pablo. 2006. Historia y Ciencias Sociales, p.105.

32 Mendizábal, María y Riffo, Jorge. 2013. "Historia, Geografía y Ciencias Sociales", p. 106; Landa del Rio, Luis y Pinto, Victoria. 2017. "Historia, Geografía y Ciencias Sociales", p. 227.

${ }^{33}$ Mendizábal, María y Riffo, Jorge. 2013. Historia, Geografía y Ciencias Sociales, p. 106; Cembrano, Dina y Cisternas, Luz. 2002. Historia y Ciencias Sociales. $2^{\circ}$ Medio. Texto del Estudiante, Santiago de Chile: Editorial Zig-Zag S. A., p. 101.

34 Donoso, María Loreto; Valencia, Lucía; Palma, Daniel y Álvarez, Rolando. 2003. "Historia y Ciencias Sociales", p. 112; Milos, Pedro; Almeyda, Liliam y Whipple, Pablo. 2006. "Historia y Ciencias Sociales", p. 105.
} 
de liderazgo, en el mundo político como civil, tanto para construir el bien común, como para cobijar aspiraciones personales de poder que promueven el enfrentamiento por sobre la colaboración entre los grupos sociales.

Dicha vinculación ética, aunque tiene implicancias para el desarrollo moral personal de cualquier líder, apunta a la conexión sustancial que debe existir entre democracia y gobierno. Aquello incluye reconocer la utilización que se hace del modelo democrático moderno para intentar compatibilizar procesos de acumulación de poder en determinadas élites dirigentes con proyectos sustentados en algún bien común genérico. Ciertamente, frente a esto, se requieren definiciones que establezcan mejores reglas para favorecer efectivamente a todos los grupos. No obstante, aquello ha ido menos aparejado con una deliberación en torno a los valores y principios de convivencia social que también son necesarios. Este aspecto, que hunde sus raíces no solo en el debate político sino en el sustrato cultural de las sociedades, incluye el cultivo de valores como la igualdad y la justicia social, y el desarrollo de prácticas de colaboración que respeten la diversidad natural entre los diversos grupos sociales, y superen los márgenes del individualismo centrado en el propio interés de estos grupos ${ }^{35}$. Consecuentemente, los contenidos sobre la vida de Bernardo O'Higgins que aportan los textos escolares constituyen un punto de partida para discutir este aspecto menos considerado respecto a las formas de gobierno y liderazgo requeridos actualmente, y que debieran apuntar fundamentalmente al fortalecimiento de la vida democrática más allá del Estado.

\section{El liderazgo político de O’Higgins en perspectiva global}

Las orientaciones para la Formación Ciudadana en Chile han ido progresivamente introduciendo la vinculación entre ciudadanía, participación y globalización ${ }^{36}$. En ese sentido, la noción de ciudadano global ha ido ampliando las definiciones sobre el buen ciudadano que habían estado constreñidas a los límites de la nación y que influenciaban los alcances para formar ciudadanos. Aparentemente, esto estaría en consonancia con los planteamientos de algunos autores respecto a preparar a los estudiantes para que desarrollen vinculaciones más allá de las fronteras y colaboren con ciudadanos de otros países en desafíos globales, al mismo tiempo que utilizan los emergentes canales de participación y comunicación entre ciudadanos de distintas nacionalidades ${ }^{37}$. No obstante, las políticas de Formación Ciudadana se han tendido a limitar a presentar la globalización como nuevo contexto en el que deberán desenvolverse los futuros ciudadanos, enfatizando los retos/riesgos que representa las nuevas demandas económicas, culturales y ecológicas que inciden en el desarrollo del país.

\footnotetext{
35 Touraine, Alain. 1998. What is democracy? Boulder, Colorado, Westview Press.

36 Ministerio de Educación. 2013. Orientaciones técnicas y guiones didácticos para fortalecer la formación ciudadana. $7^{\circ}$ Básico a $4^{\circ}$ Medio, 2013.

37 Myers, John. 2016. "Charting a democratic course for global citizenship education: Research directions and current challenges", Education Policy Analysis Archives, Vol. 24, N. 55, Tempe, Arizona, p. 1 - 15.
} 
Consecuentemente, no hay una mención explícita al protagonismo de la ciudadanía chilena en estos retos, cooperando en proyectos de integración a nivel latinoamericano o incluso, liderando iniciativas solidarias o de justicia social con ciudadanos de otras latitudes.

Por contrapartida, las referencias a Bernardo O'Higgins en los textos escolares dejan constancia del alcance internacional de su liderazgo, el que se vincula a otros líderes de la época pertenecientes a otras repúblicas nacientes en el continente ${ }^{38}$. Más aún, junto a José de San Martin, lidera al Ejército Libertador en una empresa regional que superó las fronteras coloniales /nacionales establecidas por la Corona ${ }^{39}$. Ya en el gobierno, O'Higgins procuró establecer relaciones con Estados europeos a fin de lograr el reconocimiento internacional de la independencia y de la instauración de la nueva república; y para solicitar un empréstito en Inglaterra para paliar los gastos que resultaron de la guerra independentista, lo que tuvo serias consecuencias económicas adversas para el país y para su propio gobierno ${ }^{40}$. Finalmente, vive la última etapa de su vida exiliado en el Perú, lo que habla nuevamente de su vinculación con otras ciudadanías regionales ${ }^{41}$. Todas estas experiencias permiten la discusión respecto a la vinculación que los líderes chilenos han establecido con las autoridades de otros países; de la calidad de los lazos de cooperación que se han formado y los efectos que aquello va teniendo en las ciudadanías más allá de los beneficios que puedan suponer para los gobiernos de turno.

Desde esta perspectiva, la discusión sobre el liderazgo de O’Higgins, en perspectiva global, debiese también remitir al proceso de formación del Estado nación en relación con sus Estados vecinos. En efecto, los Estados en América Latina se construyeron en función de los Estados económicamente más desarrollados, lo que significó que sus dirigentes articularan los proyectos nacionales con el propósito -generalmente no alcanzado- de integrarse al creciente capitalismo global. Esto implicó, igualmente, el establecimiento de relaciones de competencia entre los países de la región en vistas a sus propios fortalecimientos, lo que, a su vez, provocó el distanciamiento entre ellos en relación con sus procesos de desarrollo ${ }^{42}$. Frente a este contexto, el compromiso de O'Higgins con un proyecto de liderazgo cuya vinculación con las potencias del momento pasó -al menos idealmente- por establecer un bloque independiente integrado mancomunadamente por las nuevas repúblicas, es un aspecto sugerente presente en los textos escolares, que contribuye a reflexionar sobre los tipos de liderazgos que se requieren en América Latina para fortalecer los lazos entre sus ciudadanías, no sólo promoviendo lazos de colaboración, sino contribuyendo a la superación de las restricciones

\footnotetext{
38 Landa del Rio, Luis y Pinto, Victoria. 2017. "Historia, Geografía y Ciencias Sociales", p. 216.

${ }^{39}$ Millán, Hugo, Muñoz, Gabriela, Osses, Mauro y Valenzuela, Alejandra. 2019. Historia, Geografía y Ciencias Sociales. Texto del Estudiante, Santiago de Chile, Ediciones SM, p. 160.

40 Donoso, María Loreto; Valencia, Lucía; Palma, Daniel y Álvarez, Rolando. 2003. "Historia y Ciencias Sociales".

41 Müller, Javiera; Valdés, Magdalena y Caracci, María Soledad. 2009. "Historia y Ciencias Sociales”, p. 117.

42 Faletto, Enzo. 2014. “La especificidad del Estado en América Latina”, en Estudios, №31, Córdoba, pp. 205 - 236.
} 
impuestas por el Estado nación por medio de la instauración de relaciones verdaderamente transfronterizas.

\section{Comentarios finales: O’Higgins en el entrecruce entre la enseñanza de la historia y la ciudadanía}

A lo largo de este trabajo, hemos intentado proponer un marco de referencia para sugerir la vinculación entre el estudio de la vida de Bernardo O’Higgins y la Formación ciudadana, tal como se proponen en el currículo escolar. Al respecto, planteamos que, ante la ausencia relativa de contenidos que apunten a la formación para el liderazgo o para discutir respecto de formas de liderazgo que sean coherentes con la aspiración democrática del país, y su manera de interactuar con otros países en contexto de globalización, la figura de O’Higgins puede resultar relevante como referente original de un modo de ser líder y gobernar en Chile. No obstante, se debe advertir que la pregunta sobre esta relevancia remite finalmente a discusiones adicionales respecto a si el análisis sobre la Independencia de Chile, como evento fundacional, tiene valor e influencia en un contexto de Bicentenario en donde los múltiples conflictos y demandas de la ciudadanía requieren de nuevas respuestas y desafíos. Al respecto, la historiografía puede dar orientaciones importantes en momentos en que, en materia de investigaciones referidas a la vinculación entre formación ciudadana y enseñanza de la historia, de por si escasas, éstas han estado más centradas en la enseñanza de eventos de la historia reciente que a la enseñanza de eventos más distantes en el tiempo ${ }^{43}$.

Si bien en los apartados anteriores, se propusieron una serie de temáticas que podrían ser de importancia para establecer la vinculación entre Bernardo O'Higgins y la formación de ciudadanos -en buena medida ausente en las actuales directrices ministeriales- éstas sólo constituyen algunas de las conexiones que se podrían establecer. Por dicha razón, habrá que seguir profundizando para establecer con mayor claridad cuáles podrían ser esos temas más relevantes para los estudiantes o que podrían generar el tipo de discusión en el aula que promueve actitudes deliberativas ciudadanas. Más aún, habrá otras conexiones que hacer que excedan los marcos establecidos por las directrices sobre formación ciudadana que hemos considerado en este trabajo. Por ejemplo, el conflicto, como principio de análisis, tiende a ser omitido en esta área de la formación ${ }^{44}$. No obstante, el conflicto supone el entrecruce entre una serie de perspectivas que conviven en la sociedad y sobre las cuales los estudiantes

\footnotetext{
43 Magendzo, Abraham y Toledo, María Isabel. 2009. "Educación en derechos humanos: curriculum historia y ciencias sociales del segundo año de enseñanza media, subunidad régimen militar y transición a la democracia", en Estudios Pedagógicos, Vol. 35, N¹, Valdivia, pp. 139 - 154; Vásquez, Gabriela, Sánchez, María, Vásquez, Nelson y Muñoz, Carlos. 2018. "La transición democrática chilena ¿ proceso pactado o brusca ruptura?: una mirada desde la perspectiva de los estudiantes secundarios", en Tempo \& Argumento, Vol. 10, №24, Florianápolis, pp. 247 - 278. ${ }^{44}$ Banaji, Shakuntala. 2008. "The trouble with civic: a snapshot of young people's civic and political engagements in twenty-first-century democracies", Journal of Youth Studies, Vol. 11, № 5, London, pp. 543 - 560.
} 
debiesen discutir. Al respecto, la misma figura de Bernardo O'Higgins refleja que el liderazgo y el poder suponen opciones de graves consecuencias. Así, mientras O'Higgins debió asumir dichas opciones en un contexto bélico y violento, las nuevas generaciones también tienen el desafío de asumir el costo de ser líder en un Chile en constante enfrentamiento y polarización.

Asimismo, lo anterior entronca con una reflexión sobre los textos escolares que, si bien no eran objeto de este estudio, aportan a un análisis que es importante considerar. En particular, la paradoja de que un mayor foco en las habilidades de los estudiantes para un mejor aprendizaje de la historia, introducido en los textos escolares de historia chilenos para intentar superar modelos de enseñanza memorísticos ${ }^{45}$, podría estar traduciéndose, también, en una disminución de contenidos historiográficos que también son importantes para enseñar la historia en vistas a la comprensión de los fenómenos actuales. Al menos en parte, este aspecto deberá ser subsanado por los profesores que, finalmente, son quienes seleccionan y complementan los contenidos para adecuarlos a sus estudiantes. Consecuentemente, ellos podrían incorporar aspectos de la vida de O'Higgins que, teniendo resonancias en las actuales problemáticas, no se consideran en los textos escolares. Así, su condición de hijo ilegítimo, su vinculación con el pueblo mapuche, su origen de provincia, y su condición de inmigrante con la que termina sus días, son todos temas vigentes en las discusiones sobre inmigración, descentralización regional, clasismo o racismo cultural que son elementos constitutivos de las relaciones políticas en el país y que, ciertamente, inciden en los liderazgos que se ejercen ${ }^{46}$.

No obstante, la reflexión sobre Bernardo O'Higgins tampoco debiese limitarse a estudiar su vida en cuanto experiencia histórica única, sino en cuanto caso que entronca con otros tantos, y con los cuales, en último término, configuran una suerte de genealogía de liderazgos en la historia del país, que contribuyen a comprender de mejor manera las implicancias que tiene vivir en un colectivo llamado nación dirigido por un Estado, cuya relación con la ciudadanía, siempre será problemática dado los aspectos coercitivos y selectivos que involucra. Desde esa perspectiva, tal como ya se señaló anteriormente, es evidente que todo estudio sobre liderazgo debe contextualizarse bajo miradas de largo plazo que permitan la comprensión de su naturaleza a partir del desarrollo concreto que ha tenido en la sociedad ${ }^{47}$. Este aspecto, a su vez, tiene implicancias para continuar reflexionando sobre las mejores estrategias para acercar a los estudiantes al estudio de la historia, y a quienes contribuyeron a la construcción de las sociedades desde los presentes que les tocó vivir ${ }^{48}$. Desde esta perspectiva, este trabajo no se limita a una propuesta sobre cómo enseñar a O’Higgins, sino que aporta a la búsqueda

\footnotetext{
45 Sáez-Rosenkranz, Isidora. 2017. "La enseñanza de la historia en los libros de texto de educación básica en Chile", en Enseñanza de las Ciencias Sociales, 2017, Vol. 16, Barcelona, pp. 27 - 40.

${ }^{46}$ Rubilar, Luis. 2009. "Francisco de Miranda, Simón Bolívar y Bernardo O’Higgins, el Libertador de Chile", en Contextos: Estudios de Humanidades y Ciencias Sociales, Vol. 21, Santiago, pp. 63 - 72.

47 Araujo. 2016. "El miedo".

48 Wineburg, Sam. 2001. "Historical Thinking".
} 
de modos de enseñar la historia que involucre la participación de los estudiantes y signifique el desarrollo de habilidades que les conecte reflexiva y críticamente con el pasado, y les comprometa con el mundo social y político del ahora ${ }^{49}$.

Finalmente, es importante recalcar, que este análisis acerca de las contribuciones que el estudio sobre Bernardo O'Higgins podría aportar a la formación ciudadana de los estudiantes, en ningún caso se ha tratado de idealizar su figura, ni cómo ésta ha sido transmitida históricamente en el sistema escolar; como una mera extensión de los intentos por parte de las élites de solventar su modelo particular de Estado- nación ${ }^{50}$. Tampoco se trata de asignarle atributos democráticos que tienen valor en la actualidad, pero que ciertamente no estuvieron presentes en su ascensión al poder más bien restringido a su elitismo y autoritarismo político ${ }^{51}$. Sin embargo, aun reconociendo las limitaciones que desde el hoy puedan visualizarse en el actuar de O’Higgins, las formas de liderazgo y de ciudadanía global actualmente vigentes resultan de procesos históricos de construcción que siempre remiten a un pasado que no debe despreciarse en vistas a adquirir miradas más integrales que reconozcan tanto errores como avances, permitiendo visualizar perspectivas nuevas ${ }^{52}$. Ante ello, será fundamental promover la discusión entre los estudiantes ante un Bicentenario que requiere de liderazgos nuevos, pero que no serán tales, si no se sustentan en una mirada profunda respecto de quienes nos han liderado hasta el momento y de cuánto han contribuido a quienes podríamos ser.

\section{Referencias citadas}

Advisory Group on Citizenship. 1998. Education for citizenship and the teaching of democracy in schools, London, Qualifications and Curriculum Authority.

Araujo, Kathya. 2016. El miedo a los subordinados. Una teoría de la autoridad, Santiago de Chile, LOM.

Banaji, Shakuntala. 2008. "The trouble with civic: a snapshot of young people's civic and political engagements in twenty-first-century democracies", en Journal of Youth Studies, Vol. 11, N 5, Berlin, pp. 543-560.

\footnotetext{
49 Van Boxtel, Carla y van Drie, Jannet. 2018. "Historical reasoning".

50 Gatica, Enrique, González, Fabián y Navarro, Danixa. 2013. "La narrativa histórica oficial y el fantasma del héroe nacional en el aprendizaje histórico", en Revista de Pedagogía Crítica Paulo Freire, Vol. 12, № 14, Santiago, pp. 79 $-97$.

51 Ossa, Juan Luis. 2014. "The army of the Andes: Chilean and Rioplatense politics in an age of military organisation, 1814-1817", Journal of Latin American Studies, Vol. 46, Cambridge, pp. 29 - 58; Ossa, Juan Luis. 2016. "El gobierno de Bernardo O'Higgins visto a través de cinco agentes estadounidenses, 1817-1823", en Revista Co-herencia, Vol. 13, № 25, pp. 139 - 166, Medellín, pp. 139 - 166.

52 Cavieres-Figueroa, Eduardo. 2008. "Historia y bicentenario: ¿ilusiones o realidades? La necesidad de considerar la historia", en Parentini, Luis Carlos (comp.). Historiadores chilenos frente al bicentenario, Santiago de Chile: Universidad Católica Silva Henríquez.
} 
Carretero, Mario. 2007. Documentos de Identidad. La construcción de la memoria histórica en un mundo global, Buenos Aires, Paidós.

Cavieres Fernandez, Eduardo. 2019. “Urban high school students' experiences of participation as a counter-story constellation to institutional and policy guidelines in Chile", en Urban Education, pp. 1-12.

Cavieres-Figueroa, Eduardo. 2008. "Historia y bicentenario: ¿ilusiones o realidades? La necesidad de considerar la historia", en Parentini, Luis Carlos (comp.), Historiadores chilenos frente al bicentenario, Santiago de Chile, Universidad Católica Silva Henríquez.

Comisión Formación Ciudadana. 2004. Informe Comisión Formación Ciudadana, Santiago de Chile, Ministerio de Educación.

Dahl, Robert. 1988. On democracy, New Haven, Yale University Press.

Dalton, Russel. 2014. Citizen politics: public opinion and political parties in advanced industrial societies, London, SAGE.

División de Educación General. 2016. Orientaciones para la elaboración del plan de formación ciudadana, Santiago de Chile, Ministerio de Educación.

Endacott, Jason, Dingler, Matt y O’Brien, Joe. 2020. “To What Purpose? The ends and means of history education in the modern world", en Berg, Christopher, y Christou, Theodore (Eds.) The Palgrave Handbook of History and Social Studies Education, Cham, Springer, pp. 541-573.

Faletto, Enzo. 2014. "La especificidad del Estado en América Latina”, en Estudios, º 31, Córdoba, pp. 205-236.

Gatica, Enrique, González, Fabián y Navarro, Danixa. 2013. “La narrativa histórica oficial y el fantasma del héroe nacional en el aprendizaje histórico", en Revista de Pedagogía Crítica Paulo Freire, Vol. 12, N 14, Santiago, pp. 79-97.

Goldberg, Tsafrir y Savenije, Geerte. 2018. "Teaching controversial historical issues”, en Metzger, Scott y Harris, Lauren M. (Eds.) The Wiley International Handbook of history teaching and learning, New York, Willey Blackwell, pp. 503-526.

González, Fabian, Cerri, Luis y Rosso, Ademir. 2016. “Héroes y cultura histórica entre estudiantes en Chile", en Revista Brasileira de Historia, Vol. 36, N71, Sao Paulo, pp. 2-24.

Guerrero, Cristian y Cárcamo, Ulises. 2013. “Bernardo O’Higgins, entre izquierda y derecha. Su figura y legado en Chile: 1970-2008”, en Cuadernos de Historia, Vol. 39, Santiago de Chile, pp. 113-146.

Heater, Dereck. 2004. Citizenship. The civic ideal in world history, politics and education, New York, Manchester University Press.

Henn, Matt y Foard, Nick. 2014. "Social differentiation in young people's political participation: the impact of social and educational factors on youth political engagement in Britain", en Journal of Youth Studies, Vol. 17, N³ 3, pp. 360-380.

Lévesque, Stephane. 2008. Thinking historically: Educating students for the twenty-first century, Toronto, University of Toronto Press. 
Lévesque, Stephane y Clark, Penney. 2018. "Historical thinking: Definitions and educational applications", en Metzger, Scott. A. y Harris, Lauren M. (Eds.) The Wiley International Handbook of history teaching and learning, New York, Willey Blackwell, pp. 117-148.

Magendzo, Abraham y Toledo, María Isabel. 2009. “Educación en derechos humanos: curriculum historia y ciencias sociales del segundo año de enseñanza media, subunidad régimen militar y transición a la democracia", en Estudios Pedagógicos, Vol. 35, №1, Valdivia pp. 139-154.

McAvoy, Paula y Hess, Diana. 2013. "Classroom deliberation in an era of political polarization", en Curriculum Inquiry, Vol. 43, № 1, Toronto, pp. 14-47.

McLaughlin, Terence. 1992. "Citizenship, diversity and education, a philosophical perspective", en Journal of Moral Education, Vol. 21, № 3, pp. 235-250.

Ministerio de Educación. 2015. Bases curriculares. $7^{\circ}$ básico a $2^{\circ}$ medio. Chile, Santiago.

Ministerio de Educación. 2013. Orientaciones técnicas y guiones didácticos para fortalecer la formación ciudadana. $7^{\circ}$ Básico a $4^{\circ}$ Medio, Santiago de Chile, Unidad Técnico Pedagógica. Muñoz Labraña, Carlos, Martínez Rodríguez, Rosendo y Muñoz Grandón, Carlos. 2016. "Percepciones del estudiantado sobre la política, los partidos políticos y las personas dedicadas a la política al finalizar la educación secundaria en Chile", en Revista Electrónica Educare, Vol. 20, $N^{\circ} 1$, Heredia, pp. 1-16.

Myers, John. 2016. "Charting a democratic course for global citizenship education: Research directions and current challenges", en Education Policy Analysis Archives, Vol. 24, N. 55, pp. 1-15. Mycock, Andrew y Tonge, Jonathan. 2012. "The party politics of youth citizenship and democratic engagement", en Parliamentary Affairs, Vol. 65, London, pp. 138-161.

Nordgren, Kenneth y Johansson, Maria. 2015. "Intercultural historical learning: A conceptual framework", en Journal of Curriculum Studies, Vol. 47, N¹, Londres, pp. 1-25.

Ní Mhurchú, Aoileann. 2014. "Citizenship beyond the state sovereignty", en Isin, Engin y Nyers, Peter (eds.). Routledge handbook of global citizenship studies, New York, Routledge.

Ossa, Juan Luis. 2014. "The army of the Andes: Chilean and Rioplatense politics in an age of military organisation, 1814-1817", en Journal of Latin American Studies, Vol. 46, Cambridge, pp. 29-58.

Ossa, Juan Luis. 2016. “El gobierno de Bernardo O'Higgins visto a través de cinco agentes estadounidenses, 1817-1823". Revista Co-herencia, Vol. 13, № 25, Medellín, pp. 139-166.

Romero, Luis Alberto. 2009. "Enfrentar al enano nacionalista: una mirada a los libros de texto", en Temas de Historia Argentina y Americana, Vol. 14, Buenos Aires, pp. 197-214.

Ross, E. Wayne. 2017. Rethinking Social Studies: Critical Pedagogy in Pursuit of Dangerous Citizenship, Charlotte, Information Age Publishing, Inc.

Rubilar, Luis. 2009. “Francisco de Miranda, Simón Bolívar y Bernardo O’Higgins, el Libertador de Chile", en Contextos, Estudios de Humanidades y Ciencias Sociales, Vol. 21, Santiago, pp. 63-72. Sáez-Rosenkranz, Isidora. 2017. "La enseñanza de la historia en los libros de texto de educación básica en Chile”, en Enseñanza de las Ciencias Sociales, Vol. 16, Barcelona, pp. 27-40. 
Seixas, Peter. 2017. "Historical consciousness and historical thinking", en Carretero, Mario, Berger, Stefan y Grever, Maria (Eds.), Palgrave handbook of research in historical culture and education, Londres, Palgrave Macmillan, pp. 59-72.

Smith, Peter. 2012. Democracy in Latin America. Political change in comparative perspective, New York, Oxford University Press.

Stoker, Gerry. 2006. Why democracy matters. Making democracy work, New York, Palgrave MacMillan.

Touraine, Alain. 1998. What is democracy?, Boulder, Colorado, Westview Press.

Van Boxtel, Carla y van Drie, Jannet. 2018. "Historical reasoning: Conceptualizations and educational applications", en Metzger, Scott. A. y Harris, Lauren M. (Eds.) The Wiley International Handbook of history teaching and learning, New York, Willey Blackwell, pp. 149176.

Vásquez, Gabriela, Sánchez, María, Vásquez, Nelson y Muñoz, Carlos. 2018. “La transición democrática chilena ¿ proceso pactado o brusca ruptura?: una mirada desde la perspectiva de los estudiantes secundarios", en Tempo \& Argumento, Vol. 10, № 24, Florianópolis, pp. 247278.

Villalón Gálvez, Gabriel. 2021. “Conocimiento histórico y formación ciudadana. Inclusión de otras narrativas históricas para una Nueva Historia Escolar", en REIDICS. Revista de Investigación en Didáctica de las Ciencias Sociales, N8, Cáceres, pp. 89-105.

Villalón Gálvez, Gabriel, Cavieres Fernández, Eduardo y Vásquez Leyton, Gabriela. 2020. "Vinculando la enseñanza de la independencia nacional con temas socialmente relevantes para la formación de ciudadanos", en Sophia Austral, №26, Punta Arenas, pp. 33-50.

Wineburg, Sam. 2001. Historical Thinking and other unnatural acts. Charting the future of teaching the past, Filadelfia, Temple University Press.

\section{Textos escolares consultados}

Cembrano, Dina y Cisternas, Luz. 2002. Historia y Ciencias Sociales. $2^{\circ}$ Medio. Texto del Estudiante, Santiago de Chile, Editorial Zig-Zag S. A.

Donoso, María Loreto, Valencia, Lucía, Palma, Daniel y Álvarez, Rolando. 2003. Historia y Ciencias Sociales. $2^{\circ}$ Educación Media, Santiago de Chile, Santillana del Pacífico S.A. de Ediciones.

Landa del Rio, Luis y Pinto, Victoria. 2017. Historia, Geografía y Ciencias Sociales. $8^{\circ}$ básico. Texto del Estudiante, Santiago de Chile, Ediciones SM Chile S.A.

Mendizábal, María y Riffo, Jorge. 2013. Historia, Geografía y Ciencias Sociales. $2^{\circ}$ Medio Editorial. Texto del Estudiante, Santiago de Chile, Ediciones SM Chile S.A.

Milos, Pedro, Almeyda, Liliam y Whipple, Pablo. 2006. Historia y Ciencias Sociales. $2^{\circ}$ Medio. Texto para el Estudiante, Santiago de Chile, MN Editorial Ltda.

Millán, Hugo, Muñoz, Gabriela, Osses, Mauro y Valenzuela, Alejandra. 2019. Historia, Geografía y Ciencias Sociales. Texto del Estudiante, Santiago de Chile, Ediciones SM. 
Müller, Javiera, Valdés, Magdalena y Caracci, María Soledad. 2009. Historia y Ciencias Sociales. $2^{\circ}$ Educación Media. Texto para el Estudiante, Santiago de Chile, Santillana del Pacífico S.A. de Ediciones. 